\title{
Clinical Features and Socio - Demographic Factors Affecting the Prevalence of Enteric Fever: A Prospective Observational Study
}

Sadia Saber*a, Md. Tarek Alam ${ }^{\mathrm{b}}$, Nazi Yasminº, Mohammad Monwer Hossain ${ }^{\mathrm{d}}$, Rafi Faria Alam ${ }^{\mathrm{d}}$

\begin{abstract}
:
Background: Enteric fever otherwise called Typhoid fever is a systemic disease caused by Salmonella typhi and Salmonella paratyphi, having reservoir in contaminated water and food. It affects considerable number of populations of the world but regions of South Asia are most commonly affected.
\end{abstract}

Aims and Objective: The objective of this study to find the prevalence of Typhoid fever with respect to age, gender, marital status, occupation, seasonal variation, treatment of drinking domestic water source, and clinical features.

\begin{abstract}
Methodology: This is a cross- sectional observational study conducted at Bangladesh Medical College Hospital (BMCH), Dhanmondi, Dhaka from January to December 2019. Those presenting both outpatient departments or admitted to the inpatient units with complains of fever and abdominal pain underwent blood culture for the diagnosis of Typhoid fever. Patients with informed consent and positive blood culture report (including both Salmonella typhi and Salmonella paratyphi) were included in the study.

Results: Total 1375 tests were performed, out of which 407 came out to be positive blood culture report (including both Salmonella typhi and Salmonella paratyphi). Out of these tests $31.65 \%$ were males and $27.35 \%$ were females. Highest occurrence of typhoid fever was observed among males of age group 18 - 45 years and least with patients between age group above 75 years. As for the influence of occupation, male civil servants recorded the highest occurrence in the hospitals while artisans recorded the least. The influence of marital status revealed the highest occurrence in the single males with the married females had the least. In relation to months the prevalence was the highest in the month of July - September (11.42\%). Water quality, vaccination and season have great impact on the burden of typhoid fever in population. Fever, diarrhea and abdominal pain are the major symptoms associated with typhoid fever.
\end{abstract}

Conclusion: Socio-demographic factors such as age, gender, marital status, and seasonal variation, treatment of drinking water supply, occupation and presenting complaints influenced the distribution pattern of typhoid fever among the population in BMCH, Dhanmondi, Dhaka.

Keywords: Enteric Fever, Salmonella typhi and Salmonella paratyphi.

\section{INTRODUCTION:}

Typhoid fever is caused by the bacteria Salmonella typhi (S. typhi) and Salmonella paratyphi. ${ }^{(1-4)}$ This bacterium invades many organs and gives rise to symptoms on entering the blood

a. Assistant Professor of Medicine, Bangladesh Medical College \& Hospital, Dhanmondi, Dhaka.

b. Professor \& Head of the Dept. of Medicine, Bangladesh Medical College \& Hospital, Dhanmondi, Dhaka.

c. Assistant Professor of Gynae \& Obs., International Medical College $\&$ Hospital, Tongi, Gazipur.

d. Medical Officer Dept. of Medicine, Bangladesh Medical College \& Hospital, Dhanmondi, Dhaka.

*Corresponding Author:

Dr. Sadia Saber

MBBS, FCPS (Medicine), MRCP (UK), MRCP (Ireland). MRCP (Glasgow) Assistant Professor

Department of Medicine, Bangladesh

Medical College and Hospital

House: 56, Road no: 16 (new), Dhanmondi, Dhaka-1209

E-mail: sadiasaber201477@gmail.com

Cell : + 8801745898396 stream. It reaches the intestinal tract and is excreted in the feces of infected person and thus, stool cultures are used for its diagnoses. It takes one to two weeks for symptoms to develop and remains threatening for four to six weeks with headache, fever, splenomegaly, abdominal pain, relative bradycardia, and leucopenia. Patient can also experience generalized body aches, constipation, diarrhea, poor appetite and if left untreated, can cause intestinal perforation or bleeding. The reasons for these differences in disease severity are not known but may be related to differences in health care facilities, host immune responses; genetic factors in the strains of Salmonella species.

Blood culture is still used as a gold standard test for typhoid fever. ${ }^{5}$ Previously, Widal test was the sole determinant but due to its non - specificity, time consumption, and inaccuracy it is not used anymore. ${ }^{6}$ The sensitivity of blood culture is highest in the first week of the illness and reduces with advancing illnesses. The organisms may be recovered from bloodstream at any stage of the illness, but are most commonly found during the first 7 to 10 days and during relapses. Blood culture is the method of choice and has the great advantage over culture from the faeces, urine or bile. It is showing not only that patient is infected with the bacillus but that the infection 
is active. Though it is gold standard, the yield of blood culture is quite variable. In the untreated patient, blood culture is usually positive in about $80.0 \%$ during first week and declining 20 to $30.0 \%$ later in the course of the disease. ${ }^{5}$ Sensitivity of cultures can be affected by antibiotic treatment of the patient, inadequate sampling, type of culture medium, lengths of incubation, and variations of bacteremia in the patients. In addition, Salmonella cultures take 4 to 7 days for isolation and identification of the organisms. Adequate volumes of medium should be used in blood culture system to avoid negative results. A study finding suggested that $50 \mathrm{ml}$ of medium was adequate for $8 \mathrm{ml}$ of blood, presumably because of very low degrees of bacteremia in some patients.

If whole blood is to be cultured, it is essential to prevent bactericidal effects of serum either by adequate dilution of the sample in an adequate medium volume or by inhibition of serum bactericidal factors. Sodium polyanethol sulfonate (SPS) and bile salt inhibit this bactericidal effect.$^{7}$ The SPS in concentration of $0.025 \%$ to $0.03 \%$ is the best anticoagulant for blood. It is also anticomplementary and antiphagocytic, and interferes with the activity of some anti-microbial agents, notably amino glycosides. Taking samples of blood on several occasions may improve the results of culture. Three types of blood cultures have been in use such as traditional or conventional blood culture, lysis centrifugation and automated blood culture. ${ }^{(5-7)}$

The existing estimate of the global burden of typhoid fever is 16 million illnesses and 600,000 deaths annually. ${ }^{7}$ Typhoid fever poses a considerable burden to the 5.5 billion people living in low and middle income countries. ${ }^{8}$ In 2000, it was estimated that over 2.6 million episodes of typhoid fever occurred worldwide resulting in 216,000 deaths and among them Asians were the major sufferers, with $90 \%$ of morbidity and mortality. ${ }^{(1,2,9)}$

Typhoid fever is endemic in the subcontinent, Southeast Asia, Middle East and South and Central America, with hyperendemicity reported in Pakistan. ${ }^{10}$ The accurate incidence and prevalence of typhoid fever is not available but it ranks second in the prevalence of South Asia. The main threat in Bangladesh especially Dhaka is poor hygienic values, unclean water supply, and underlying socioeconomic conditions. ${ }^{1-4}$ Contaminated water and food are the main reservoirs for S. typhi and patients get infected by typhoid fever via ingestion. ${ }^{11,12}$

The aim of the current research is to find the relation between typhoid fever and other factors such as time (month of year), age, gender, occupation, marital status and other socioeconomic variations.

\section{Materials and Methods}

This is a cross - sectional observational study conducted at Bangladesh Medical College Hospital, Dhanmondi, Dhaka .The study was conducted for a limited period of 12 months in which 1375 patients were selected both outpatient departments or admitted to the inpatient units with complaints of fever, headache and abdominal pain.

Inclusion Criteria are a) Patients with positive blood culture report (including both Salmonella typhi and Salmonella paratyphi), b) Patients aged 18 years and above,c)patients who gave informed consent. Patients already on antibiotics and patients and patients who gave repeated samples of blood culture were excluded from the study.

Patients visiting outpatient departments or admitted to the inpatient units with the complaints of fever, headache and abdominal pain were registered for the study. 1375 study subjects were registered. A short questionnaire was developed and was tested by conducting a pilot study. Variables like age, gender, marital status, occupation, seasonal variation, treatment of drinking water and clinical features were included in the questionnaire and participants' blood samples were taken to conduct blood cultures. $10 \mathrm{ml}$ of blood were collected and was transferred to the site laboratory facility within $4 \mathrm{~h}$ of sample collection at ambient temperature. An isolation rate of $92 \%$ of blood culture with the Bactec 460 Radiometric system using a blood: broth ratio of 1:6 was found. Where blood cultures do not provide a definite diagnosis and fever persists for 7 or more days, additional serology based tests may be performed as clinically indicated but these will not contribute to the estimates of typhoid incidence. $S$. Typhi isolated from blood culture contributes to confirmed typhoid fever estimates. All typhoid isolates will undergo antimicrobial susceptibility testing and will be archived for future genomic characterization. All other significant culture isolates including S. Paratyphi were documented.

This research work was approved by the ethical review committee of BMCH Laboratory (IERB No: 2018/Dec/01) with permission of the head of institution. Participants were described briefly for the study objectives and procedure. Informed consent was taken and interviews were conducted in privacy. Counseling was done after interview; participants were given no additional benefits and were free to withdraw from the survey at any point.

Data was entered in Excel 2010 then analyzed in software of IBM Statistical Package for the Social Sciences (SPSS) Statistics for Windows, Version 21.0. (IBM Corp, Armonk, NY). Frequency and percentages were reported for the variables and Chi square analyses were conducted to determine association of blood culture positivity with all the variables. P-Value $<0.05$ was considered statistically significant.

\section{RESULT:}

During the period of 12 months, total 1375 blood culture tests were performed out of which 407 (29.6\%) tests were blood culture positive for enteric fever.As shown in Table 1, prevalence of typhoid fever was found more in males than in females with significant difference $(p=0.04)$ (Table 1). 
Table 1

\begin{tabular}{|c|c|c|c|c|}
\hline Gender & $\begin{array}{c}\text { Total number } \\
\text { of tests } \\
\text { performed }\end{array}$ & $\begin{array}{r}\text { Blo } \\
\text { Cul } \\
\text { Positiv }\end{array}$ & $\begin{array}{l}\text { od } \\
\text { ture } \\
\text { ve tests }\end{array}$ & $P$ - value \\
\hline & \multicolumn{2}{|c|}{ Frequency } & \multicolumn{2}{|l|}{ Percentage } \\
\hline Male & 717 & 227 & $31.65 \%$ & \\
\hline Female & 658 & 180 & $27.35 \%$ & 0.04 \\
\hline Total & 1375 & 407 & $29.6 \%$ & \\
\hline
\end{tabular}

In this study, participants were grouped into different groups according to age. Typhoid was mostly detected in participants of age group of 18-45years $(\mathrm{p}=0.03)$ (Table 2)

Table 2

\begin{tabular}{lccc}
\hline $\begin{array}{l}\text { Age } \\
\text { (years) }\end{array}$ & $\begin{array}{c}\text { Total number } \\
\text { of tests } \\
\text { performed }\end{array}$ & $\begin{array}{c}\text { Blood } \\
\text { Culture } \\
\text { Positive tests }\end{array}$ & P - value \\
\hline
\end{tabular}

\begin{tabular}{lrccl}
\hline & \multicolumn{3}{c}{ Frequency } & Percentage \\
$\mathbf{1 8 - 4 5}$ & 710 & 265 & 19.27 & \\
$\mathbf{4 6 - 7 5}$ & 480 & 97 & $7.05 \%$ & 0.03 \\
$>\mathbf{7 5}$ & 185 & 45 & $3.27 \%$ & \\
Total & $\mathbf{1 3 7 5}$ & $\mathbf{4 0 7}$ & $\mathbf{2 9 . 6 \%}$ & \\
\hline
\end{tabular}

Fig 1 shows influence of Seasonal variation on Typhoid Fever Prevalence

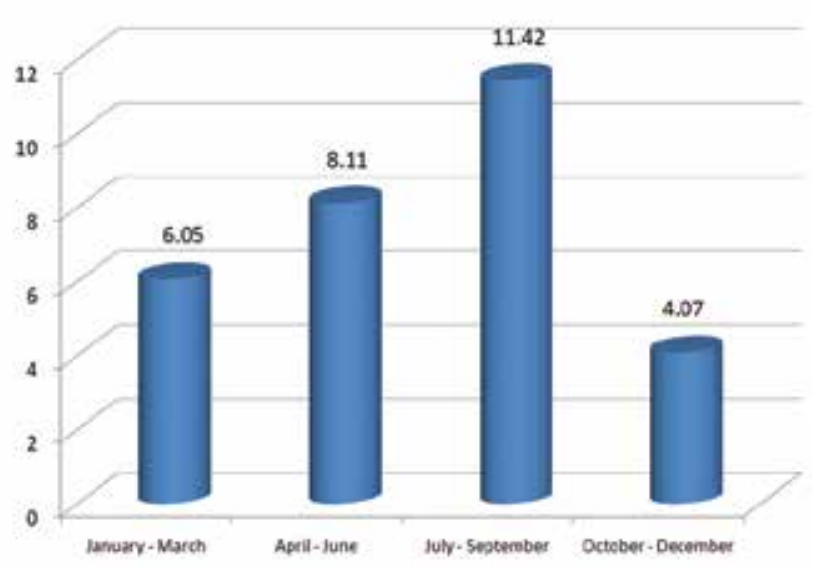

Fig 1

Table 3 shows Influence of Marital Status on Typhoid Fever Prevalence in the Study Population
Table 3

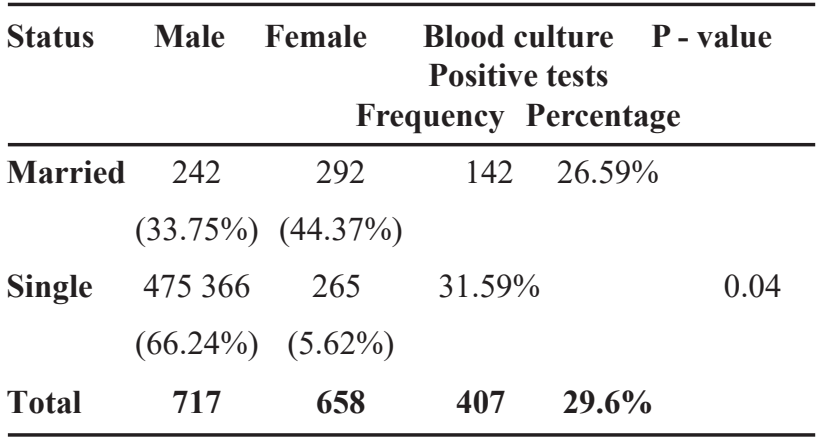

Majority of the population (45\%) did not take any measure to treat water before drinking; only $31 \%$ of them used boiling as a method of water treatment and $24 \%$ population used chlorination followed by boiling (Fig 2)

\section{Distribution of cases according to treatment of water for drinking}

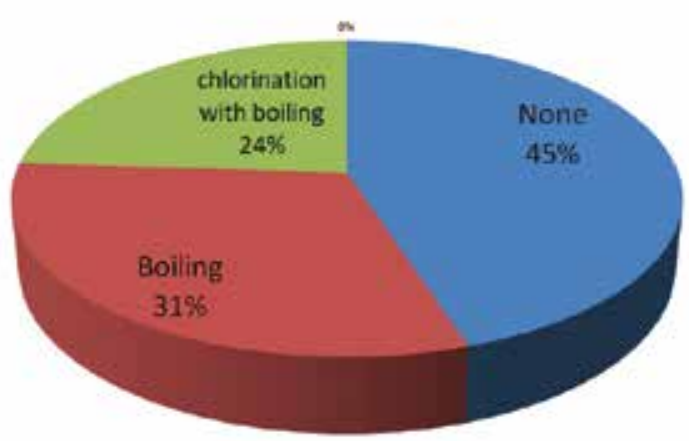

Figure 2

Table 4 shows Influence of Occupation on Typhoid Fever Prevalence in the Study population.

Table 4

\begin{tabular}{lrcc}
\hline Occupation & Number & $\begin{array}{r}\text { Blood Culture Positive tests } \\
\text { Frequency }\end{array}$ & Percentage \\
\hline Civil Servants & 465 & 210 & $51.59 \%$ \\
Farmers & 75 & 10 & $2.45 \%$ \\
Artisans & 97 & 35 & $8.59 \%$ \\
Traders & 171 & 41 & $10.07 \%$ \\
Students & 257 & 44 & $10.81 \%$ \\
House wives & 310 & 67 & $16.46 \%$ \\
Total & 1375 & 407 & $29.6 \%$ \\
\hline
\end{tabular}


Fig 3 shows distribution of Study Population according to presenting complaints.

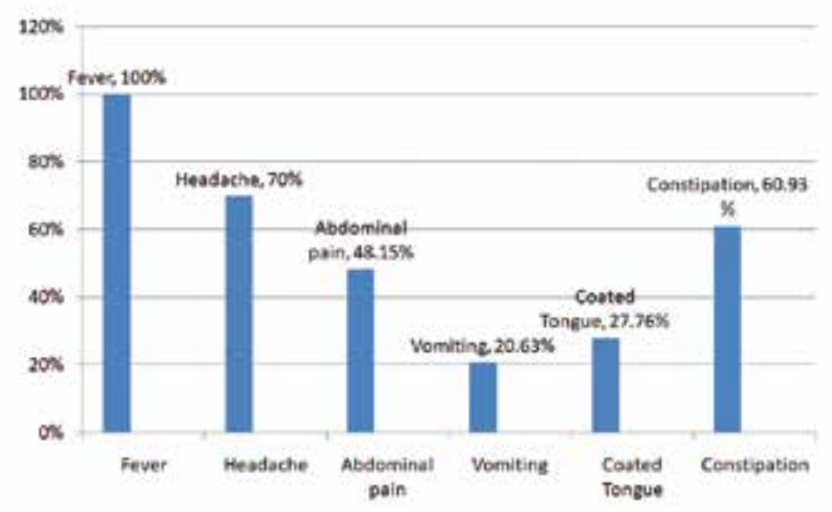

Fig 3

\section{DISCUSSION:}

Gender wise, males were found to be more prominently affected than females which might be due to the fact that males are the earning members of the family spending most of the time out of home, and so making him liable to eat outside home. In a study conducted in Karachi, it was reported that about $9.1 \%$ of food handlers were positive for Salmonella. ${ }^{13}$

Month of July, August and September are season changing months in Dhaka during which typhoid organism grows with boost, because these conditions are favorable for organism growth. This might be the reason why blood culture positive results were more during this month in this study. However; further studies need to be conducted to check the prevalence throughout the year.

Table 2 shows the age distribution pattern of typhoid fever. The age of 16 to 45 years (38.09\%) showed the highest positive result while the least positive result was found in the age group of 75 and above (17.65\%). This finding can be presumed to be so due to the carefree, sanitary and feeding attitudes of young adults of this age group. This concurs with findings of Esohe et al ${ }^{14}$ who reported 66 patients out of 173 patients who tested typhoid fever positive to be within the range of 26 to 35 years. Older adults are presumably more respondent to sanitary feeding conditions and relatively resistant due to frequent boosting of immunity ${ }^{15}$.

Table 3 shows the influence of marital status on the prevalence of typhoid fever. The highest typhoid positive result is shown in single patients $(31.59 \%)$. The reason why single patients are more affected by typhoid fever than married patients is not known as marriage demands makes it mandatory for a sizeable number of them to cook in their homes paying close attention to basic hygienic practices. ${ }^{16}$

Knowledge about modes of transmission and prevention of enteric fever among people were very poor. A low socioeconomic status is reflective of poor standards of living, ignorance, illiteracy and poor personal hygiene, increasing the susceptibility to infections like enteric fever Although, a direct link between domestic water treatment and typhoid fever infection may be difficult to establish, possible fecal contamination of most water sources could be a major contributory factor to the high infection frequency. However most individuals strongly believe that stream is safe for drinking even when untreated but when underground contamination from burst sewer pipes gets high, chances of water contamination and infection eventually rise. ${ }^{16}$ Majority of the population $(45 \%)$ did not take any measure to treat water before drinking; only $31 \%$ of them used boiling as a method of water treatment and $24 \%$ population used chlorination followed by boiling. This view has been supported by different other studies conducted previously in Dhaka, Jakarta and Darjeeling. ${ }^{17-19}$

Table 4 shows the influence of occupation on the prevalence of typhoid fever. The highest blood culture positive result is shown in civil servants $(51.59 \%)$. The high occurrence rate of typhoid fever observed among civil servants could be traced to their living habits. It is noticeable that most civil servants patronize nearby eateries for their lunch while some buy hawked foods, most of which are not hygienically prepared. Though individuals with other occupations indulge in same act, the rate seems higher in students as well. A similar study reported that Civil Servants were the occupation with the second highest blood culture positive results which was also traced to their living habits as reported by Ajayi et al ${ }^{16}$.He also found civil servants to be the most susceptible occupation ( $41.2 \%$ occurrence).

Clinical presentations of enteric fever is nonspecific classical step ladder pattern of fever is not commonly found in all patient group. In our study the different clinical manifestations among the patients who were positive in the Blood culture was seen (Fig 3). Fever was the most constant symptom representing $407(100 \%)$, Headache was present in $287(70 \%)$, abdominal pain in $196(48.15 \%)$, coated tongue in $113(27.76 \%)$, vomiting in $84(20.63 \%)$, diarrhoea in 263 (64.62\% not shown in the figure) and constipation was seen in $248(60.93 \%)$ cases. Our findings were similar according to Bienvenido et al. in which Fever was the most constant symptom in their study, Headaches and chills were present in $63 \%$, abdominal pain $48 \%$, anorexia in $44 \%$, malaise in $33 \%$, vomiting in $31 \%$ and diarrhoea in $49 \%$. Constipation and nausea were seen in $12 \%$ and disorientation in $3 \%$ of patients. ${ }^{20}$

This study generated the data on prevalence of typhoid fever among patients having fever, headache and abdominal pain. This study focused on different factors including gender, age, occupation, marital status and seasonal variation. By this study, population at risk and month of maximum cases were identified. The number of typhoid fever cases can be reduced by targeting affected population during the most vulnerable time of the year.

\section{CONCLUSION:}

From the result of this study, it can be concluded that socio-demographic factors studied influenced the distribution pattern of typhoid fever among the populace in Dhanmondi, 
Dhaka. Enlightenment programs on basic rules of hygiene should be encouraged among the people while public health agencies should base plans for control of the disease on the predisposing factors revealed by this study such as quality of drinking water. These findings have a huge implication on the implementation of typhoid control measures and highlight the need of intensive educational campaigns to ensure adherence to the practice of personal hygiene in general. As a public health preventive measure, intensive community health education needs to be integrated into the typhoid fever control measures in Dhaka, Bangladesh.

\section{References:}

1. Mweu E, English M. Typhoid fever in children in Africa. Trop Med Int Health. 2008; 13:532-540

2. Malisa A, Nyaki H. Prevalence and constraints of typhoid fever and its control in an endemic area of Singida region in Tanzania: Lessons for effective control of the disease. J Public Health Epidemiol. 2010;2:93-99.

3. Vaishnavi C, Kochhar R, Singh G, Kumar S, Singh S, Singh K. Epidemiology of typhoid carriers among blood donars and patients with biliary, gastrointestinal and other related diseases. Microbiol Immunol. 2005; 49:107-112.

4. Kariuki S. Typhoid fever in sub-Saharan Africa: Challenges of diagnosis and management of infections. J Infect Dev Ctries. 2008; 2:443-447.

5. Wain J, Diep TS, Bay PV, Walsh AL, Vinh H, Duong NM, et al. Specimens and culture media for the laboratory diagnosis of typhoid fever. J Infect Dev Ctries. 2008; 2:469-474.

6. Somerville PC, Lewis M, Koornhof HJ, Alberts M, Alberts HW, Raymond R. The Widal test in the diagnosis of typhoid fever in the Transvaal. S Afr Med J. 1981;59:851-854.

7. Crump JA, Luby SP, Mintz ED. The global burden of typhoid fever. Bull World Health Organ. 2004; 82:346-353.

8. Antillon M, Warren JL, Crawford FW, Weinberger DM, Kurum E, Pak GD, et al. The burden of typhoid fever in low and middleincome countries: A meta-regression approach. PLoS Negl Trop Dis. 2017;11:e0005376.
9. Acosta CJ, Danovaro-Holliday MC, Baiqing D, Bhattacharya SK, Agtini MD, Bhutta ZA, et al. A study of typhoid fever in five Asian countries: disease burden and implications for controls. Bull World Health Organ. 2008; 86:260

10. Hayat AS, Shah SIA, Shaikh N. Study for evaluation of Typhidot (IgM) in early and rapid diagnosis of typhoid fever at tertiary care settings in Abbottabad. Professional Med J. 2011; 18:259-264.

11. Farooqui A, Khan A, Kazmi SU. Investigation of a community outbreak of typhoid fever associated with drinking water. BMC Public Health. 2009; 9:1.

12. Kanungo S, Dutta S, Sur D. Epidemiology of typhoid and paratyphoid fever in India. J Infect Dev Ctries. 2008;2:454-460.

13. Siddiqui TR, Bibi S, Mustufa MA, Ayaz SM, Khan A. High prevalence of typhoidal Salmonella enterica serovars excreting food handlers in Karachi-Pakistan: A probable factor for regional typhoid endemicity. J Health, PopulNutr. 2015; 33:27.

14. Esohe, AI, Osazua, F, Ajayi, SA, Ebueku, A, Igbinigbe, IF. Dual Infection with Typhoid and and Malaria in Febrile Patients in Ikare akoko, Nigeria. Int. J. Trop. Med.2012; 7(1): 49-52.

15. Parry, CM. Epidemiological and clinical aspects of human typhoid fever: In Salmonella' Infections: Clinical, Immunological and Molecular Aspects, ed. Mastroeni P, Maskell D. Cambridge University Press, 2005; pp. 1-10.

16. Ajayi OE, Olukunle, OF, Boboye, BE. Prevalence of Typhoid Fever among Different Socio-demographic Groups in Ondo State, Nigeria. Journal of Applied Life Sciences International, 2015; 3(2): $89-95$.

17. Ram PK, Naheed A, Brooks WA, Hossain MA, Mintz ED, Breiman RF. Risk factors fortyphoid fever in a slum in Dhaka, Bangladesh. Epidemiol Infect 2007; 135(3):458-65.

18. Vollaard AM, et al. Risk Factors for Typhoid and Paratyphoid Fever in Jakarta, Indonesia. JAMA 2004; 291(21): 2607-15.

19. Sharma PK, Ramkrishnan R, Hutin Y, Manickam P, Gupte MD. Risk factors for typhoid in Darjeeling, West Bengal, India: evidence for practical action. Tropical Medicine and International Health 2009; 14(6): 696-702.

20. Bienvenido DA, Pamela L (1980) Enteric fever: A review of ninety cases. Phil J Microbiol Infect Dis 9: 118-128. 